\title{
A levitated magnetic dipole configuration as a compact charged particle trap
}

\author{
H. Saitoh ${ }^{1,2}$, M. R. Stoneking ${ }^{2,3}$, and T. Sunn Pedersen ${ }^{2,4}$ \\ ${ }^{1}$ Graduate School of Frontier Sciences, The University of Tokyo, Kashiwa, Chiba 277-8561, Japan, \\ ${ }^{2}$ Division E4, Max Planck Institute for Plasma Physics, Greifswald 17491 and Garching 85748, Germany, \\ ${ }^{3}$ Department of Physics, Lawrence University, Appleton, Wisconsin 54911, USA, \\ ${ }^{4}$ Institute of Physics, The University of Greifswald, Greifswald 17489, Germany *
}

(Dated: March 10, 2020)

\begin{abstract}
As a magnetic confinement configuration for electron-positron pair-plasmas, the APEX collaboration [T. Sunn Pedersen et al., New J. Phys. 14, 035010 (2012)] plans to construct a compact levitated dipole experiment with a high-temperature superconducting coil. In order to realize stable levitation of the dipole field coil, a simple feedback-controlled levitation system was constructed with conventional analogue circuits. We report the properties of a prototype levitation system using a permanent magnet and compare its behavior to predictions from a stability analysis. We also present a practical review needed for the construction of a compact levitated dipole trap system based on [J. Morikawa et al., TEION KOGAKU, (J. Cryo. Soc. Jpn.) 39, 209 (2004)]. Numerical orbit analysis suggests improved confinement properties of charged particles in a dipole field trap by replacing the permanent magnet with a levitated superconducting coil magnet. Such a compact dipole field configuration is potentially applicable to the confinement of various charged particles including positrons and electrons.
\end{abstract}

\section{INTRODUCTION}

After studies of plasma confinement in internal conductor devices in 1970's [1], experiments in a levitated dipole configuration, or a laboratory magnetosphere [2], are being carried out that focus on the relaxation states of plasmas suitable for advanced fusion concepts. The Ring Trap (RT) project of The University of Tokyo was motivated by efficient plasma confinement observed in the Jovian magnetosphere [3], which is explained by the theory of self-organization of flowing plasmas $[4,5]$. The basic concept of dipole confinement was investigated in Proto-RT [6, 7], where the dipole field was generated by a mechanically supported copper coil. In order to minimize perturbations to plasmas caused by the mechanical support structures of the coil, a levitated high-temperature superconducting coil was developed and used in the Mini-RT experiment [8]. Based on these basic studies, RT-1 was constructed and started operation in 2006 [2]. In RT-1, physics of both fusion-oriented high temperature plasmas and nonneutral plasmas have been investigated [9, 10]. Another approach to the advanced fusion concept in the dipole field configuration [11] has been also studied at the Levitated Dipole Experiment (LDX) [12] whose levitation system design is reported in [13].

As a confinement geometry for non-neutral plasmas [14] including antimatter plasmas [15] and other charged particles[16-19], toroidal geometries may be attractive because of their capability to confine charged particles consisting of multiple species at any non-neutrality [20, 21]. In the axisymmetric magnetic configuration of the dipole, excellent charged particle confinement properties are expected because of the conservation of the canonical angular momentum. By utilizing this property, the A Positron-Electron eXperiment (APEX) collaboration plans to use a compact levitated dipole geometry as the magnetic trapping configurations for

*saito@ppl.k.u-tokyo.ac.jp electron-positron pair-plasmas. Experimental understanding of the unique properties of the pair plasmas $[22,23]$ is the final goal of APEX. Another approach to electron-positron plasma creation is also underway using intense laser [24, 25]. Initial experiments with a mechanically-supported permanent magnet [26], operated at the NEutron induced POsitron Source MUniCh (NEPOMUC) positron facility [27], achieved lossless injection of the positron beam into the closed dipole field configuration [28] and stable trapping of positrons [29]. These experiments with the low energy positron beams of NEPOMUC [30, 31] demonstrated the excellent confinement properties of the dipole magnetic field configuration.

One of the next major milestones of the APEX project is the simultaneous confinement of both positrons and electrons in the dipole field configuration. For the stable confinement of pair-plasmas, the levitation of a dipole field coil magnet is needed, which may be realized by a feedback-controlled levitation system. In addition to its application for the realization of electron-positron plasmas, using low-energy positrons or using intense lasers, this configuration is also potentially applicable for trapping multiple charged particle species for ion and plasma physics experiments.

In this study, we developed a feedback-controlled magnetic levitation system to be used for the compact levitated dipole experiment of the APEX project. Although Earnshaw's theorem [32] forbids stable levitation of an object against gravity with static electric and magnetic fields, there are several methods to stabilize the levitation [33-38]. In section II, we review the mechanism of magnetic levitation with a feedbackcontrolled system [39-43] and consider the practical choice of the operation parameters of the levitation system for a charged particle trap. In section III, we report on a simple feedbackcontrolled levitation system realized using conventional analogue circuits [44] and analyze the stability of the system. The properties of the magnetic levitation with this system are investigated using a small permanent magnet and compared with the stability analysis. In section IV, we analyze the orbit of charged particles in a compact levitated dipole experiment 
in comparison with a permanent magnet dipole field configuration.

\section{EQUILIBRIUM AND STABILITY ANALYSIS OF MAGNETIC LEVITATION}

\section{A. Force balance for a floating magnet}

In this section, we review the levitation mechanism needed for the stability analysis and levitation experiments [41, 43]. Figure 1 shows a typical geometry of a magnetic levitation experiment. We assume that a circular floating $(\mathrm{F})$ coil or magnet is levitated at the equator $(z=0)$ of the experiment using an upward magnetic force from a circular levitation (L) coil. We anticipate coaxially placing the $\mathrm{L}$ coil above the F coil to provide an upward levitation force if the currents are in the same direction. In addition, we choose a configuration to stabilize the tilt motion of the F coil, as we will discuss later. According to the vertical position of the F coil, which is monitored by a laser positioning sensor, for example, the L coil current is adjusted with a feedback-controlled system. The $\mathrm{L}$ coil carries a total current of $I_{\mathrm{L}} N_{\mathrm{L}}$ which generates an attractive magnetic force to levitate the F coil with a mass $m_{\mathrm{F}}$ and a total current of $I_{\mathrm{F}} N_{\mathrm{F}}$. Here $I_{\mathrm{L}}$ and $I_{\mathrm{F}}$ are coil currents, and $N_{\mathrm{L}}$ and $N_{\mathrm{F}}$ are the numbers of turns in each coil. The major radius and vertical position of the $\mathrm{F}$ coil are $r_{\mathrm{F}}$ and $z_{\mathrm{F}}$. Although we perform this analysis with the levitation of a superconducting coil in mind, we take the current in the F coil to be fixed in the following analysis. While magnetic flux is conserved for a superconductor, we can chose a levitation configuration so that the expected variation in the current associated with the perturbations we consider is negligible.

The vertical motion of the F coil in this system is determined by the equation of motion,

$$
m_{\mathrm{F}} \frac{d^{2} z_{\mathrm{F}}}{d t^{2}}=-2 \pi r_{\mathrm{F}} N_{\mathrm{F}} I_{\mathrm{F}} B_{\mathrm{r}}-m_{\mathrm{F}} g
$$

where $B_{\mathrm{r}}$ is the radial component of magnetic field at the location of the $\mathrm{F}$ coil $\left(r_{\mathrm{F}}, z_{\mathrm{F}}\right)$ generated by the $\mathrm{L}$ coil. Here current in both coils is taken to be in the positive azimuthal direction (counter-clockwise when viewed from above). For a ring current, the radial component of the field may be numerically calculated using complete elliptic integrals of the first and second kinds, $K(k)$ and $E(k)$. We assume that the circular L coil of radius $r_{\mathrm{L}}$, current $I_{\mathrm{L}}$, and $N_{\mathrm{L}}$ turns is located at the vertical position of $z=z_{\mathrm{L}}$. By using $K(k)$ and $E(k)$, where

$$
k=\sqrt{\frac{4 r r_{\mathrm{L}}}{\left(r+r_{\mathrm{L}}\right)^{2}+\left(z-z_{\mathrm{L}}\right)^{2}}},
$$

the radial component of the magnetic field generated by the $\mathrm{L}$ coil at $(r, z)$ is

$$
\begin{aligned}
B_{\mathrm{r}}(r, z) & =\frac{\mu_{0} N_{\mathrm{L}} I_{\mathrm{L}}\left(z-z_{\mathrm{L}}\right)}{2 \pi r\left(\left(r+r_{\mathrm{L}}\right)^{2}+\left(z-z_{\mathrm{L}}\right)^{2}\right)^{1 / 2}} \\
& \times\left(-K(k)+\frac{r_{\mathrm{L}}^{2}+r^{2}+\left(z-z_{\mathrm{L}}\right)^{2}}{\left(r_{\mathrm{L}}-r\right)^{2}+\left(z-z_{\mathrm{L}}\right)^{2}} E(k)\right) .
\end{aligned}
$$

Because $B_{\mathrm{r}}$ is proportional to the $\mathrm{L}$ coil current $I_{\mathrm{L}}$ for a fixed geometry and fixed $N_{\mathrm{L}}$, we may write (1) as

$$
m_{\mathrm{F}} \frac{d^{2} z_{\mathrm{F}}}{d t^{2}}=-2 \pi r_{\mathrm{F}} N_{\mathrm{F}} h I_{\mathrm{F}} I_{\mathrm{L}}-m_{\mathrm{F}} g
$$

using

$$
B_{\mathrm{r}}\left(r_{\mathrm{F}}, z_{\mathrm{F}}\right)=h\left(r_{\mathrm{F}}, z_{\mathrm{F}}\right) I_{\mathrm{L}}
$$

In the equilibrium state of (4), the force balance equation is

$$
2 \pi r_{\mathrm{F}} N_{\mathrm{F}} h I_{\mathrm{F}} I_{\mathrm{L}}+m_{\mathrm{F}} g=0 .
$$

For a fixed geometry of the F coil, the upward force on the $\mathrm{L}$ coil is proportional to $B_{\mathrm{r}}$ generated by the $\mathrm{F}$ coil. This field strength profile is explained in the next subsection.

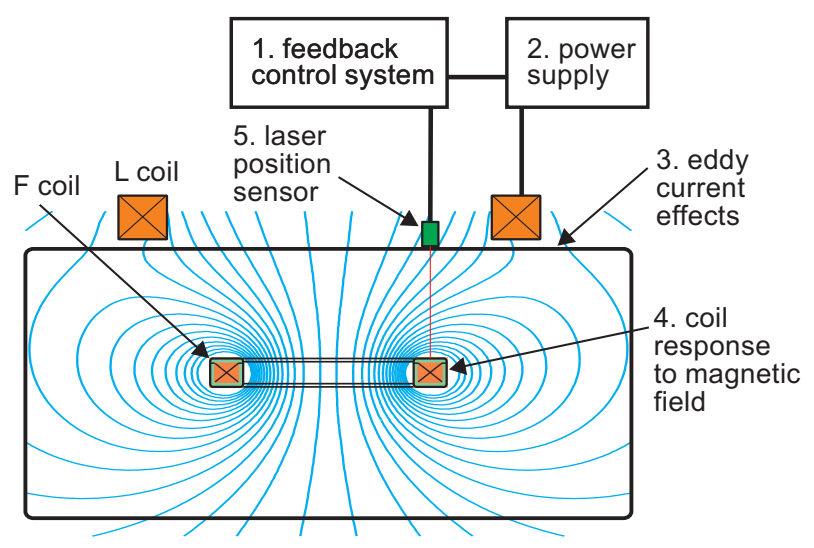

FIG. 1. A schematic of a typical levitated dipole experiment and its feedback-controlled levitation system.

\section{B. Stability of levitation}

In order to evaluate the stability of vertical coil motion, we define the inverse scale length, $\alpha$, for the change in the vertical magnetic force $F_{\mathrm{Z}}(z)$ as

$$
\alpha(z)=\frac{d F_{\mathrm{z}} / d z}{F_{\mathrm{Z}}}=\frac{d\left(I_{\mathrm{F}} B_{\mathrm{r}}\right) / d z}{I_{\mathrm{F}} B_{\mathrm{r}}} .
$$

The vertical coil motion is unstable when $\alpha$ is positive. For a permanent magnet, (7) becomes

$$
\alpha(z)=\frac{1}{B_{\mathrm{r}}} \frac{d B_{\mathrm{r}}}{d z}=\frac{1}{h} \frac{d h}{d z},
$$

assuming that the field of the magnet is generated by an equivalent ring current.

Typical oscillation frequency of the F coil is an important system parameter toward the development of a feedbackcontrolled stabilizing system. For small vertical oscillation motion of the $\mathrm{F}$ coil near the equilibrium point of $z=0$, the 

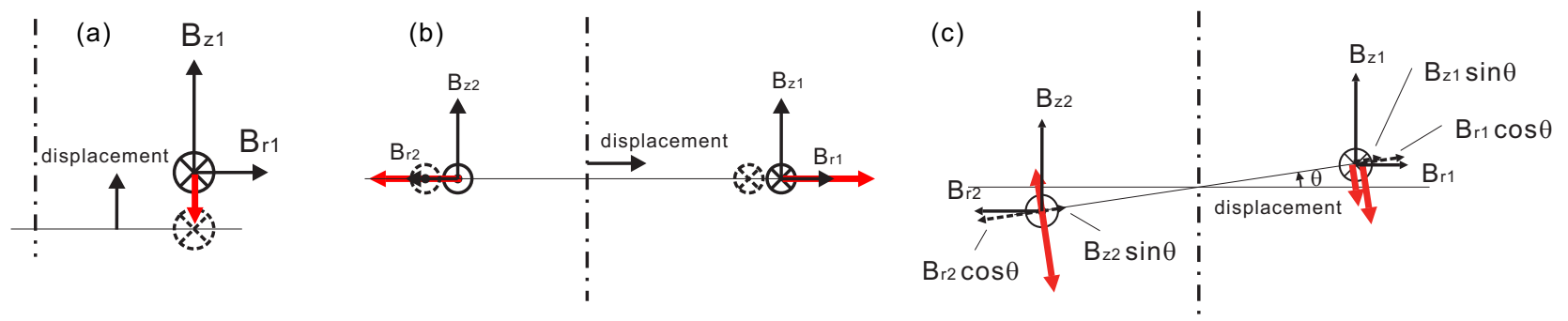

FIG. 2. When the magnetic field components are positive in the directions shown (black arrows) and the direction of the current is indicated (counter-clockwise from above), the forces on the coil to decide stabilities (red arrows) are shown for (a) vertical, (b) slide, and (c) tilt displacements of the $\mathrm{F}$ coil from the equilibrium levitation positon.

coil oscillation motion is approximated as a harmonic oscillation in a restoring force of

$$
F_{\mathrm{Z}}(z)=-k z=-2 \pi r_{\mathrm{F}} I_{\mathrm{F}} N_{\mathrm{F}} \frac{d B_{\mathrm{r}}}{d z} z
$$

The vertical oscillation frequency of the F coil is then

$$
f=\frac{1}{2 \pi} \sqrt{\frac{2 \pi r_{\mathrm{F}} I_{\mathrm{F}} N_{\mathrm{F}}}{m_{\mathrm{F}}} \frac{d B_{\mathrm{r}}}{d z}}
$$

For the parameters of a permanent magnet levitation system described below, we have $f=5 \mathrm{~Hz}$. In general, it is difficult to stabilize fast oscillations using a feedback control system. This is mainly because of the effects of delay times of the system, caused by the finite response speed of a power supply and laser positioning sensor, and eddy currents. Thus stabilization of the slower vertical motion of a larger-mass coil is easier, although the entire properties of the levitation system of course depend on many parameters in addition to the characteristic frequency.

The motions of a floating coil magnet are classified into vertical, slide, and tilt motions, as shown in Fig. 2. We review the linear stability analysis of these motions with respect to position of the $\mathrm{F}$ and $\mathrm{L}$ coils, in order to decide the operation parameters of the experiment. Variation of the magnetic force on a small coil segment due to a vertical displacement is given by

$$
\frac{d\left(N_{\mathrm{F}} I_{\mathrm{F}} B_{\mathrm{r}}\right)}{d z}=N_{\mathrm{F}} I_{\mathrm{F}} \frac{d B_{\mathrm{r}}}{d z} .
$$

again assuming that $I_{\mathrm{F}}$ is constant.

For the vertical motion shown in Fig. 2 (a), we consider the Lorentz force working at one point of the $\mathrm{F}$ coil because of the axial symmetry of the system. With a small vertical deviation $\Delta z$ of the coil position from the equilibrium point of $z=0$, the radial magnetic field at the $\mathrm{F}$ coil position at $r=r_{\mathrm{F}}$ is

$$
B_{\mathrm{r} 1}=B_{\mathrm{r} 0}+\left.\frac{\partial B_{\mathrm{r}}}{\partial z}\right|_{r=r_{\mathrm{F}}, z=0} \Delta z,
$$

where $B_{\mathrm{r} 0}$ is a value at the equilibrium point. This vertical motion is stable when

$$
0<\left.\frac{\partial B_{\mathrm{r}}}{\partial z}\right|_{r=r_{\mathrm{F}}, z=0},
$$

generating a restoring force for a positive deviation of $\Delta z$. Because $B_{\mathrm{r}}$ is generally negative in the assumed configuration, this condition means that the absolute value of $B_{\mathrm{r}}$ is a decreasing function of $z$ near $z=0$.

For the slide instability, we consider the Lorentz forces working at two symmetric points on the F coil as shown in Fig. 2 (b), again due to the symmetry of the system. With a small position deviation $\Delta r$ in a horizontal direction, the vertical magnetic field strengths at the two positions are

$$
\begin{gathered}
B_{\mathrm{Z} 1}=B_{\mathrm{Z} 0}+\left.\frac{\partial B_{\mathrm{Z}}}{\partial r}\right|_{r=r_{\mathrm{F}}, z=0} \Delta r, \\
B_{\mathrm{Z} 2}=B_{\mathrm{Z} 0}-\left.\frac{\partial B_{\mathrm{Z}}}{\partial r}\right|_{r=r_{\mathrm{F}}, z=0} \Delta r .
\end{gathered}
$$

Because the F coil current is the same at positions 1 and 2, the stability condition is simply

$$
B_{\mathrm{z} 1}<B_{\mathrm{z} 2} .
$$

From (14) and (15), the slide motion is stable when

$$
\left.\frac{\partial B_{\mathrm{Z}}}{\partial r}\right|_{r=r_{\mathrm{F}}, z=0}<0 .
$$

For the tilt motion of the F coil, as shown in Fig. 2 (c), we consider a small angular deviation from the equilibrium position and compare the Lorentz forces working at two symmetric points of the F coil. There are four force components that produce torque on the coil. They depend on the following quantities using the small angle approximation.

$$
\begin{gathered}
B_{\mathrm{r} 1} \cos \theta \sim B_{\mathrm{r} 0}+\left.\frac{\partial B_{\mathrm{r}}}{\partial z}\right|_{r=r_{\mathrm{F}}, z=0} r_{\mathrm{F}} \theta, \\
B_{\mathrm{Z} 1} \sin \theta \sim B_{\mathrm{z} 0} \theta, \\
B_{\mathrm{Z} 2} \sin \theta \sim B_{\mathrm{z} 0} \theta, \\
B_{\mathrm{r} 2} \cos \theta \sim B_{\mathrm{r} 0}-\left.\frac{\partial B_{\mathrm{r}}}{\partial z}\right|_{r=r_{\mathrm{F}}, z=0} r_{\mathrm{F}} \theta .
\end{gathered}
$$


(a)

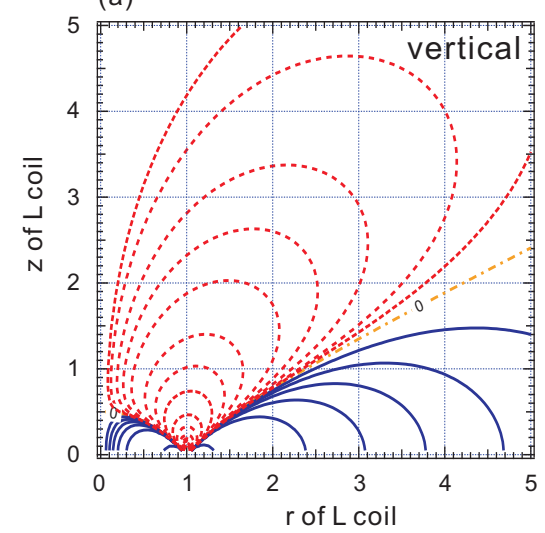

(b)

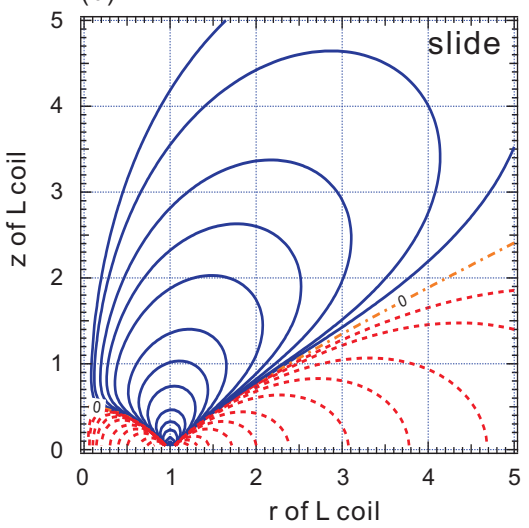

(c)

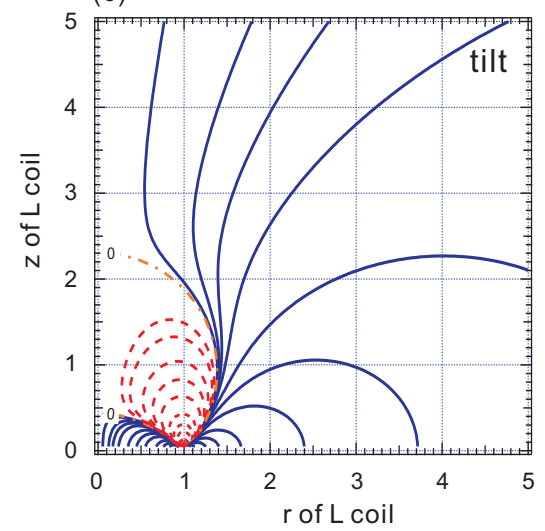

FIG. 3. Stability maps of the (a) vertical, (b) slide, and (c) tilt motions of the F coil as functions of the radius and vertical position of the L coil. The radius and vertical position of the F coil are normalized to be $r=1$ and $z=0$. Regions satisfying the conditions (13), (17), and (23) are plotted as solid blue lines, while dashed red lines show unstable regions. The chain lines in orange and labeled '0' are the stability boundaries.

The tilt motion is stabilized when

$$
B_{\mathrm{r} 2} \cos \theta<B_{\mathrm{r} 1} \cos \theta+B_{\mathrm{z} 1} \sin \theta+B_{\mathrm{z} 2} \sin \theta,
$$

which yields

$$
0<B_{\mathrm{Z} 0}+\left.r_{\mathrm{F}} \frac{\partial B_{\mathrm{r}}}{\partial z}\right|_{r=r_{\mathrm{F}, z=0}}
$$

These stability conditions solely depend on the spatial configuration of the F and L coils. We plot (13), (17), and (23) in Fig. 3 for various $L$ coil positions by fixing the position of the $\mathrm{F}$ coil at a normalized position. Namely, we assume that the $\mathrm{F}$ coil is approximated as a ring current with a radius of $r=1$ and located on the $z=0$ plane. The contours with dashed lines show the unstable regions, while regions with solid lines satisfies the stable conditions. A clear interpretation from the diagrams is that it is impossible to stabilize all of these instabilities simultaneously just by adjusting and coil configuration. This can be understood because, in a current-free region where the curl of the magnetic field is zero, the RHS of (13) equals the LHS of (17). Therefore, vertical and slide motions are not simultaneously stabilized, as shown in Fig. 3 (a) and (b). Vertical instability is one dimensional, while slide instability is two dimensional. Because the detection and control of one-dimensional motions are mush easier than those for twodimensional displacements, we chose a coil configuration so that the F coil is stable for slide motion and unstable for vertical motion. The remaining vertical instability may be stabilized by a feedback-controlled system, as explained in the next section. As can be seen from Fig. 2 (c), tilt motion can be stabilized by placing the $\mathrm{L}$ coil at a location to avoid instability. In particular, if the $\mathrm{L}$ coil radius is more than 1.4 times the radius of the $\mathrm{F}$ coil, tilt stability is assured. It is known that the tilt instability may be stabilized by using additional coils, but we will focus on a two-coil system in this study.

As well as the stability conditions, we need to consider the levitation force required to realize efficient levitation of the coil magnet, which is related to the required capacity of the

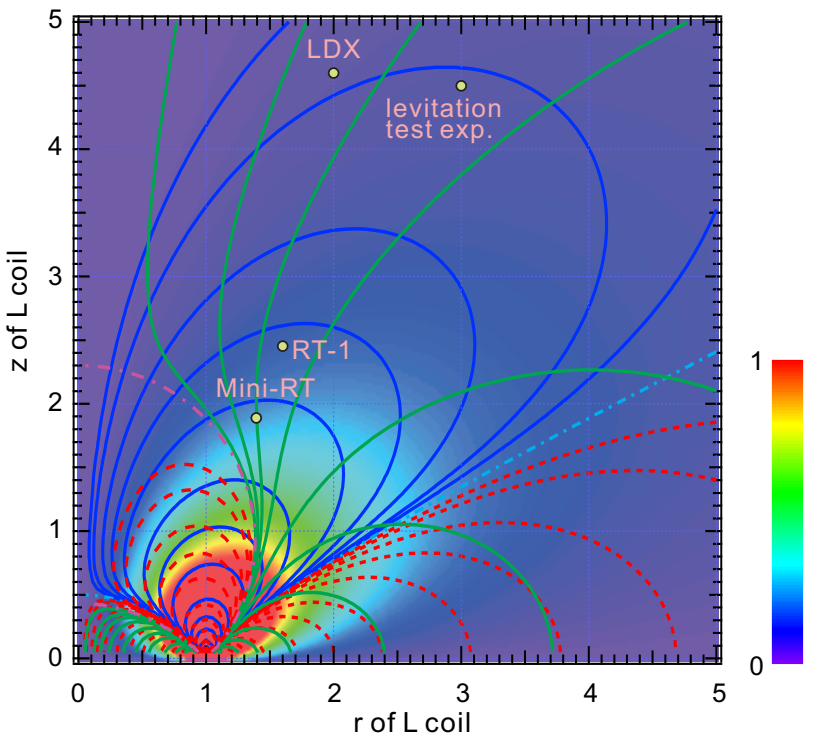

FIG. 4. Color contours show radial magnetic field $B_{\mathrm{r}}$ at the $\mathrm{F}$ coil position of $(r, z)=(1,0)$ generated by the $\mathrm{L}$ coil located at various positions. The field strength is normalized to the value at $(r, z)=(1,0.5)$. Lines shows stable (solid lines) and unstable (dashed lines) regions for slide and tilt motions of the $\mathrm{F}$ coil for various $\mathrm{L}$ coil positions and the $\mathrm{F}$ coil fixed at $(r, z)=(1,0)$.

$\mathrm{L}$ coil and power supplies. This is determined by the magnitude of the radial magnetic field generated by the $\mathrm{L}$ coil at the position of the F coil. The color contours in Fig. 4 plot $B_{\mathrm{r}}$ generated by the $\mathrm{L}$ coil at the position of the $\mathrm{F}$ coil as a function of the $\mathrm{L}$ coil position. The stabilities of slide and tilt motions are also superimposed in the figure. From these contours one can see that for a given $\mathrm{L}$ coil radius there are two vertical positions that give the same $B_{\mathrm{r}}$ (and therefore, for a given $\mathrm{F}$ coil mass and current a possible equilibrium position. One of these $\mathrm{L}$ coil positions is always in the region of slide stability (vertical instability) and the other is slide unsta- 
ble (vertically stable). The coil configuration may be decided according to these equilibrium and stability properties of the levitation system. Parameters of the several levitated dipole experiments and a levitation test experiment, to be described in the following section, are also plotted in the figure. All of the experiments shown are stable for slide and tilt motions but unstable for vertical motion.

\section{LEVITATION SYSTEM AND STABILITY ANALYSIS}

Through the analysis summarized in the previous section, it is clear that a feedback-controlled system is required to stabilize the vertical motion of the $\mathrm{F}$ coil. In this section, we construct such a system using conventional analogue circuits with a test levitation experiment using a permanent magnet. The stability of the system is analyzed using transfer functions [43] and the analysis is compared with experimental results.

\section{A. Levitation analysis and test experiment}

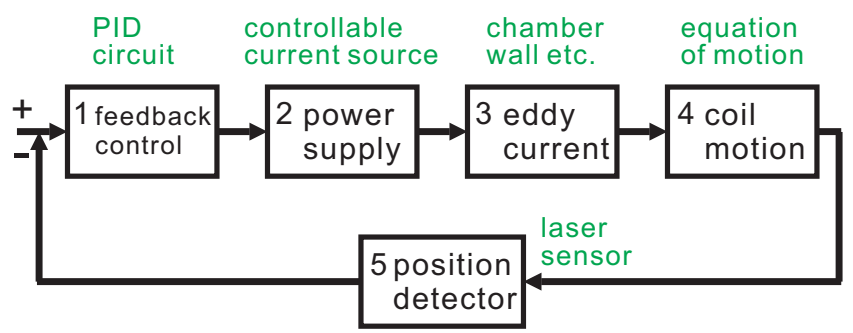

FIG. 5. Flow chart for the levitation system of a levitated dipole experiment.

Figure 5 shows the flow chart of the levitation system corresponding to Fig. 1 [43]. The position signal from a laser sensor is sent to a feedback-control circuit that produces an output signal accordingly. This output signal controls the current of the $\mathrm{L}$ coil so that the $\mathrm{F}$ coil is stably levitated. In a real experiment, there is a time lag between the $\mathrm{L}$ coil current and $B_{\mathrm{r}}$ at the $\mathrm{F}$ coil because of the eddy currents induced in conductors (e.g. a vacuum chamber). The $\mathrm{F}$ coil responds to $B_{\mathrm{r}}$ according to the equation of motion.

We investigated levitation stabilization using a simple experiment with a permanent magnet as shown in Fig. 6. A cylindrical neodymium magnet (MISUMI HXN20-3) of diameter $20 \mathrm{~mm}$, height $3 \mathrm{~mm}$, and weight $7 \mathrm{~g}$. was levitated with this system. Including buffer material wrapped around the magnet, the total weight of the magnet was $8.4 \mathrm{~g}$. Field strength at the magnet surface was $0.18 \mathrm{~T}$, according to a measurement with a Hall magnetic sensor probe. The magnetic field of this permanent magnet is approximated as a current loop of $2990 \mathrm{~A}$ and diameter of $20 \mathrm{~mm}$. The levitation coil with 90 turns was located above the magnet, as shown in the figure, and was operated at a current of $7 \mathrm{~A}$ for the equilibrium levitation point of the magnet at $z=0 \mathrm{~cm}$. The levitation coil

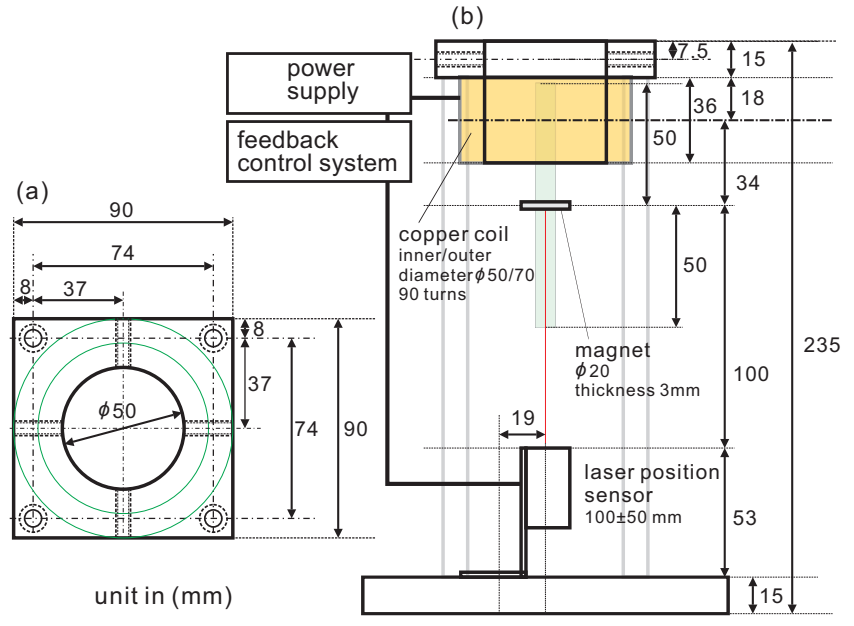

FIG. 6. A schematic of a levitation test experiment with a permanent magnet, (a) the top view and (b) side view of the experiment. The magnet is stably levitated by the magnetic force of a levitation coil whose current is feedback-controlled according to the magnet position.

was approximated as a current loop of $630 \mathrm{~A}$ and diameter of $60 \mathrm{~mm}$. The levitation coil current was supplied by a $10 \mathrm{~A}$ power supply, Elektro-Automatik PS3065-10B, whose output current was externally controlled by input voltage into its analogue interface. The vertical magnet position was monitored by a laser sensor, Sick OD-1, which can measure a distance in a range of 50 and $150 \mathrm{~mm}$ from the sensor head. This laser sensor had an analogue voltage output between 0 and $10 \mathrm{~V}$ proportional to the object's position in the measurable range.

We use a proportional-integral-derivative (PID) circuit to provide feedback to the $\mathrm{L}$ coil power supply which is made with easily available and low cost analogue operational amplifiers, as shown in Fig. 7. This circuit is based on the design of J. Morikawa [39]. We used Analog Devices OP97, lownoise small-drift operational amplifiers. At present, this is a circuit used one laser position sensor system with only one input interface. Analogue output signal form a laser position sensor is sent to U1 and U2. In future experiments, we plan to add two laser sensors, using $\mathrm{U} 2$ as an averaging circuit for the three inputs. The output signal from $\mathrm{U} 2$ is sent to a differential amplifier U3. The output voltage from U3 is proportional to the difference between the coil position signal and a reference signal $V_{\text {ref }}$. This signal $V_{\text {err }}=V_{\mathrm{i}}-V_{\text {ref }}$ is called an error signal. Here $V_{\text {ref }}$ was generated by an adjustable voltage regulator, Texas Instruments LM317. The output of the differential amplifier U3 is then sent to the P, I, and D circuits, which generates

$$
\begin{gathered}
V_{\mathrm{P}}=-\frac{R_{43}}{R_{41}} V_{\mathrm{err}}, \\
V_{\mathrm{I}}=-\frac{1}{R_{51} C_{51}} \int V_{\mathrm{err}} d t, \text { and } \\
V_{\mathrm{D}}=-R_{63} C_{61} \frac{d V_{\mathrm{err}}}{d t} .
\end{gathered}
$$




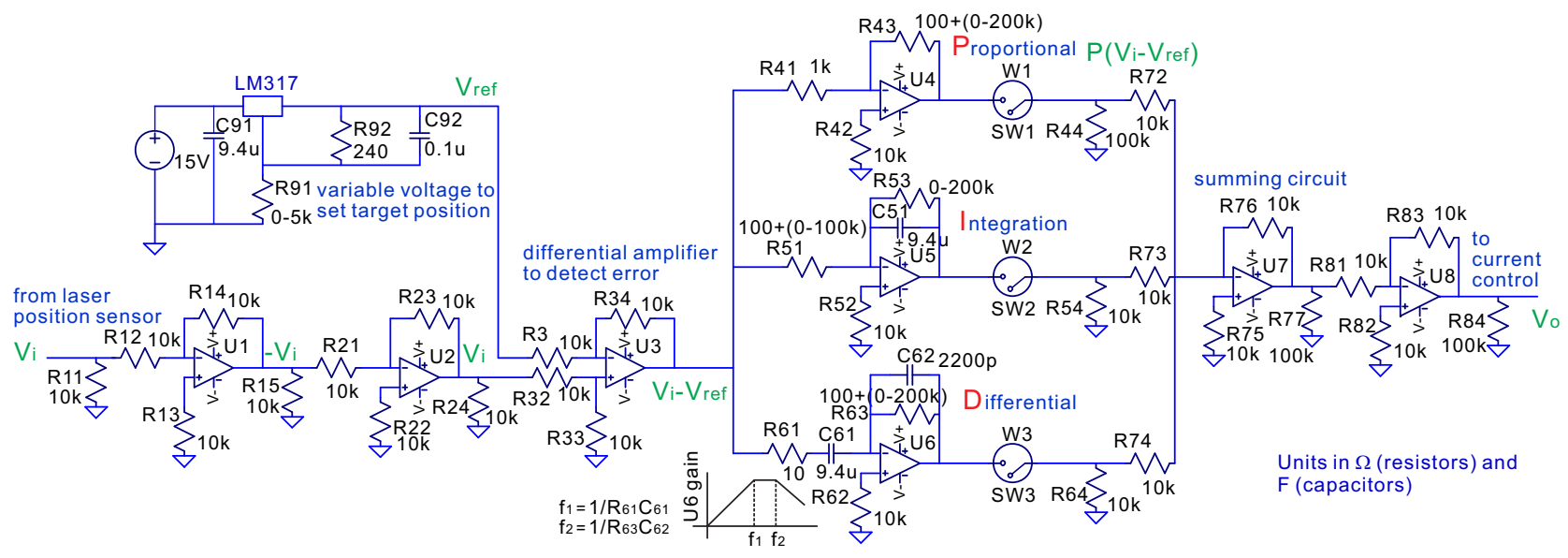

FIG. 7. A PID control system realized by conventional analog components to feedback-control the L coil current for the F coil and magnet levitation using the signal from a laser positioning sensor. U1-8 are Analog Devices OP97 with $0.1 \mu \mathrm{F}$ bypass capacitors at each power supply pins. DC voltages of $\pm 15 \mathrm{~V}$ were supplied by a series-regulated power supply.

These signals were combined by a summing circuit U7. The output voltage of inverter $\mathrm{U} 8, V_{\mathrm{O}}$, was used as a current control signal, which was sent to the control interface of the power supply of the L coil.
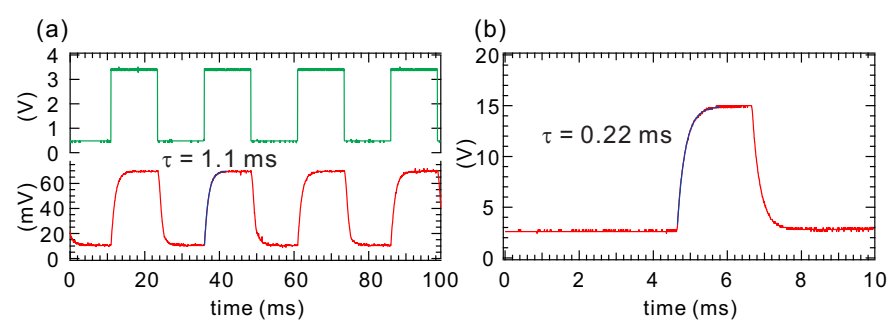

FIG. 8. (a) Time response of a system with the L coil and power supply PS3065-10B, current output signal (bottom) against the input of rectangular control voltage (top). (b) Time response of laser position sensor OD-1, against fast $(<0.1 \mathrm{~ms})$ change of a target position.

In order to stabilize the $D$ component of the circuit, around U6 in Fig. 7, the bandwidth of this amplifier was limited with the characteristic frequencies of $f_{1}=1 / R_{61} C_{61}$ and $f_{2}=$ $1 / R_{63} C_{62}$, as shown in the figure, which should be much higher than the oscillation frequency of the $\mathrm{F}$ coil motion. In deciding the value of $f_{2}$, it also should be considered that $R_{63}$ is a variable resistance. One should also try to reduce the capacitive component at the output of U6 between the ground in constructing the circuit. When the transfer length between the laser positioning system and the PID control circuit was long, we found that a conventional RC low pass filter with a time constant of $\sim 1 \mathrm{~ms}$, installed at the input of the PID circuit, was efficient in reducing the noise in the position signal. In order to avoid the switching noises, DC voltages for the circuits were supplied by a series regulator based power supply circuit.

The transfer function of each component of the PID circuit is related to the total circuit transfer function $G_{1}$ by

$$
G_{1}=P+D s+I \frac{1}{s} \sim P\left(1+\frac{D}{P} s\right)
$$

where $s=j \omega, P=R_{43} / R_{41}<200$ and $D=R_{63} C_{61}<1.88$ with parameters in Fig. 7. Here we neglect the $I$ component which is not necessarily needed for levitation and often satisfies $I / s<<P, D s$.

The time responses of the system with the $\mathrm{L}$ coil and its power supply is shown in Fig. 8 (a). For the L coil power supply system, we approximate $G_{2}$ as

$$
G_{2}=\gamma \frac{1}{1+s / 909}(\mathrm{~A} / \mathrm{V})
$$

a first-order transfer function with a $1.1 \mathrm{~ms}$ time constant, which was obtained by an exponential fitting as shown in the figure. Here $\gamma$ is the gain of the power supply, i.e., output current (A) per input voltage (V), and $\gamma=1$ in this experiment.

Eddy current effects increase the response time of the system, which also may be approximated as a first-order transfer function. However, because the test experiment has no vacuum chamber, we ignore the eddy current effects as

$$
G_{3}=1 \text {. }
$$

The equation of motion of the $\mathrm{F}$ coil is linearized by taking $I_{\mathrm{L}}=I_{\mathrm{L} 0}\left(1+I_{\mathrm{d}} / I_{\mathrm{L} 0}\right)$ and $h=h_{0}(1+\alpha z)$ as

$$
m_{\mathrm{F}} \frac{d^{2} z}{d t^{2}}=-2 \pi r_{\mathrm{F}} N_{\mathrm{F}} h_{0} I_{\mathrm{F}} I_{\mathrm{L} 0}\left(1+\frac{I_{\mathrm{d}}}{I_{\mathrm{L} 0}}+\alpha z\right)-m_{\mathrm{F}} g
$$

Because the equilibrium requires $-2 \pi r_{\mathrm{F}} N_{\mathrm{F}} h_{0} I_{\mathrm{F}} I_{\mathrm{L} 0}-m_{\mathrm{F}} g=0$, we have

$$
m_{\mathrm{F}} \frac{d^{2} z}{d t^{2}}=m_{\mathrm{F}} g\left(\frac{I_{\mathrm{d}}}{I_{\mathrm{L} 0}}+\alpha z\right)
$$




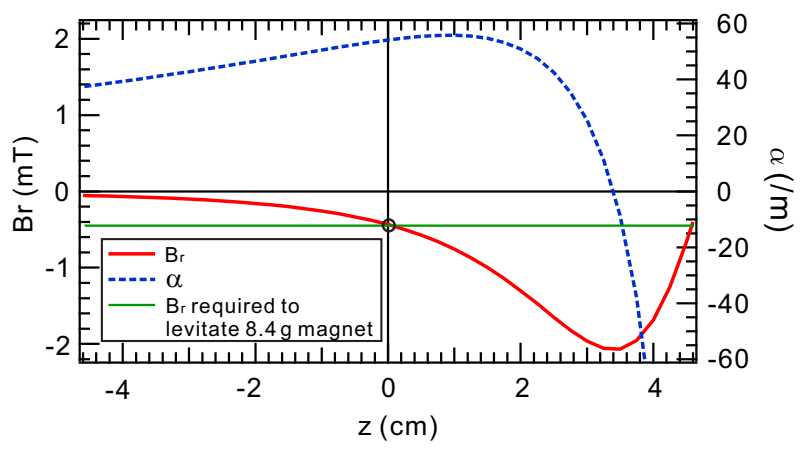

FIG. 9. The values of $B_{\mathrm{r}}$ and $\alpha$ of the levitation test experiment for different vertical positions of the magnet. The $\mathrm{L}$ coil is located at $\mathrm{z}=$ $+4.75 \mathrm{~cm}$.

By taking the Laplace transform, this equation becomes

$$
s^{2} Z(s)=\frac{g}{I_{\mathrm{L} 0}} I_{\mathrm{d}}(s)+g \alpha Z(s)
$$

Then the transfer function of the coil motion is

$$
G_{4}=\frac{Z(s)}{I_{\mathrm{d}}(s)}=\frac{1}{I_{\mathrm{L} 0} \alpha} \frac{1}{s^{2} /(g \alpha)-1} .
$$

For the present parameters, force balance is realized when the $\mathrm{L}$ coil, approximated by a ring current, is located at $z=4.75 \mathrm{~cm}$. We numerically calculate $B_{\mathrm{r}}, d B_{\mathrm{r}} / d z$, and $\alpha$, as shown in Fig. 9. Because $B_{\mathrm{r}}=0.436 \mathrm{mT}$ and $d B / d r=$ $-0.0234 \mathrm{~T} / \mathrm{m}$ at $z=0 \mathrm{~cm}$, at the position of the $\mathrm{F}$ coil, we have $\alpha=54.1 / \mathrm{m}$. Also, the $\mathrm{L}$ coil current was $I_{\mathrm{L} 0}=7 \mathrm{~A}$. For these parameters, the transfer function of the equation of motion is given by

$$
G_{4}=\frac{1}{379} \frac{1}{s^{2} / 530-1} \quad(\mathrm{~m} / \mathrm{A}) .
$$

Because the variation of $\alpha$ and $B_{\mathrm{r}}$ is relatively weak near the equilibrium point, as shown in Fig. 9, it is expected that the stability condition is also not very sensitive around $z=0$. This suggest the possibility of stable magnetic lift up and landing of the magnet by controlling the levitation coil current.

For the laser sensor, whose time responses is shown in Fig. 8 (b), we include the response time of $0.22 \mathrm{~ms}$ for the first-order transfer function and gain of 100, which yields

$$
G_{5}=100 \frac{1}{1+s / 4550} \quad(\mathrm{~V} / \mathrm{m}) .
$$

By combining $G_{1}$ to $G_{5}$ using the block diagram in Fig. 5, we have the total transfer function $G_{T}=G_{1} G_{2} G_{3} G_{4} /(1+$ $\left.G_{1} G_{2} G_{3} G_{4} G_{5}\right)$ which can be written as,

$$
G_{\mathrm{T}}=\frac{\frac{P}{379}\left(1+\frac{D}{P} s\right)\left(1+\frac{s}{4550}\right)}{\left(1+\frac{s}{909}\right)\left(\frac{s^{2}}{530}-1\right)\left(1+\frac{s}{4550}\right)+\frac{100}{379} P\left(1+\frac{D}{P} s\right)}
$$

for the entire levitation system. The characteristics equation of the system is written as, by using the characteristic polynomial, the denominator of this equation,

$$
a_{4} s^{4}+a_{3} s^{3}+a_{2} s^{2}+a_{1} s+a_{0}=0,
$$

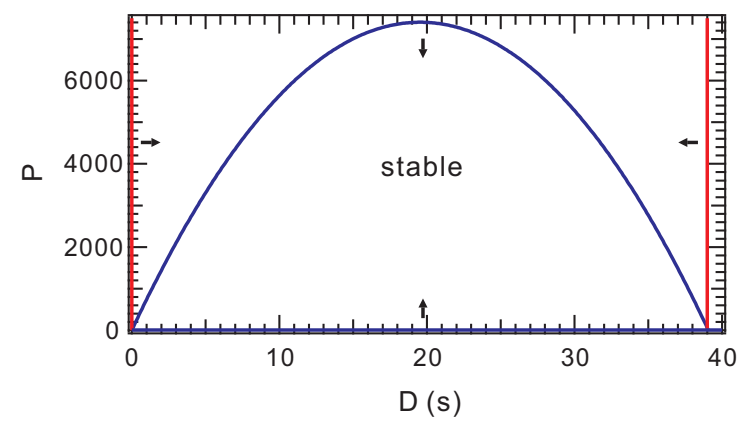

FIG. 10. Stability conditions in (41) (red lines) and in (42) (blue lines) of the levitation test experiment for various $D$ and $P$ values. The arrows indicate conditions to satisfy these equations.

where $a_{4}=4.56 \times 10^{-10}, a_{3}=2.49 \times 10^{-6}, a_{2}=1.89 \times 10^{-3}$, $a_{1}=0.264 D-1.32 \times 10^{-3}$, and $a_{0}=0.264 P-1.00$. The solutions of the characteristic equation are called poles. In order that the system is stable, the real parts of all poles must be negative. Otherwise, i.e., when the characteristic equation has a positive pole, the step response (response of a system for input of a step function) has a divergent exponential term. Here in order to determine the stability of this system, we use the Routh-Hurwitz stability criterion [45], which is equivalent to the above statement about the poles. According to this criterion, the stability conditions of this system are the following three equations.

$$
\begin{gathered}
a_{\mathrm{n}}>0, \\
\left|\begin{array}{ll}
a_{3} & a_{1} \\
a_{4} & a_{2}
\end{array}\right|=a_{3} a_{2}-a_{4} a_{1}>0, \text { and } \\
\left|\begin{array}{ccc}
a_{3} & a_{1} & 0 \\
a_{4} & a_{2} & a_{0} \\
0 & a_{3} & a_{1}
\end{array}\right|=a_{3} a_{2} a_{1}-a_{4} a_{1}^{2}-a_{3}^{2} a_{0}>0 .
\end{gathered}
$$

From these equations, we have the stability conditions of the system as follows.

$$
\begin{aligned}
& 5.00 \times 10^{-3}<D<39.0, \text { and } \\
& 3.79<P<-19.4 D^{2}+758 D .
\end{aligned}
$$

Lines in Fig. 10 shows these conditions. The vertical motion of the F coil is stabilized by choosing appropriate feedback parameters. Experimentally it is convenient to operate in the lower left region of stability where the values of $\mathrm{P}$ and $\mathrm{D}$ are simultaneously smaller.

\section{B. Levitation experiments}

By using the system described above, we tested the feedback-controlled levitation of a permanent magnet. The 
magnet was initially placed below the equilibrium point on a supporting plate at $z=-1.3 \mathrm{~cm}$, and smoothly moved up to $z=0 \mathrm{~cm}$ by adjusting the $V_{\text {ref }}$ of the control circuit. As shown in Fig. 11, the magnet was stably levitated without time constraints when the $V_{\text {ref }}, P$, and $D$ values were appropriately adjusted. At the end of the levitation, the magnet was moved down to $z=-1.3 \mathrm{~cm}$ again by adjusting the value of $V_{\text {ref }}$.

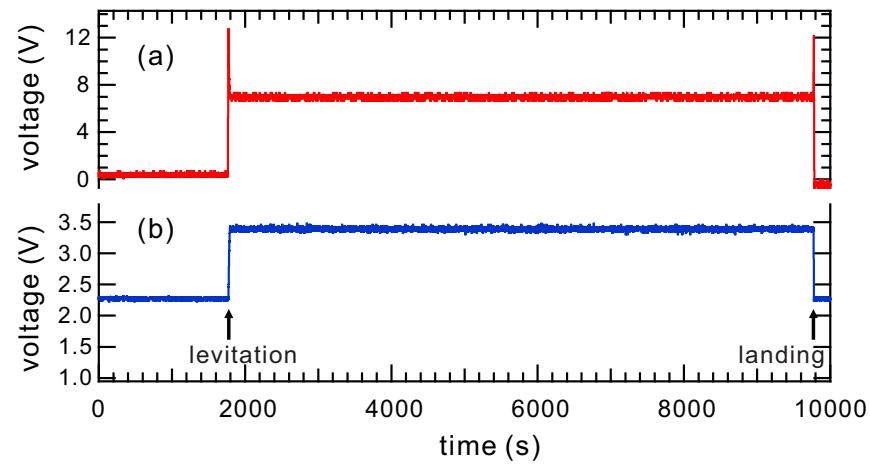

FIG. 11. Long time ( $\sim 8000 \mathrm{~s})$ levitation of the permanent magnet realized by the stabilization of its vertical motion by the feedbackcontrolled system. Signals of (a) laser sensor position output and (b) PID control circuit output.

The equilibrium levitation state is realized as follows. For a fixed equilibrium coil position, the L coil current $I_{\mathrm{L} 1}$ and position signal $V_{\mathrm{i} 1}$ from a laser sensor are fixed values. This equilibrium is decided according to the equation of motion in the gravity. Here two conditions, pertain: 1) that L coil current is sufficient to satisfy the minimum equilibrium requirements for the F coil (magnet) parameters and 2) that the values of $P$ and D satisfy (41) and (42). The DC component of the output from the the feedback circuit is $V_{\mathrm{O}}=-P\left(V_{\mathrm{i} 1}-V_{\mathrm{ref}}\right)$. The DC coil current may be controlled so that it is proportional to $V_{\mathrm{O}}$, we can write it as $-\gamma P\left(V_{\mathrm{i} 1}-V_{\text {ref }}\right)$. Then this system has a steady state solution determined by the control variables $V_{\text {ref }}$ and $P$ so that

$$
I_{\mathrm{L} 1}=-\gamma P\left(V_{\mathrm{i} 1}-V_{\mathrm{ref}}\right)
$$

is satisfied. The $\mathrm{F}$ coil is levitated at a certain point according to the values of $V_{\text {ref }}$ and $P$. According to the velocity of the levitated magnet, the $D$ component of the circuit provides a stabilization force on the magnet. It is noted that we can realize an equilibrium solution with a same $I_{\mathrm{L}}$ value and vertical position with various set of $V_{\text {ref }}$ and $P$ values. We confirmed this relation in (43) with experiments. In the present experimental setup, we have $\gamma=1$. In Fig. 12, we plot values of $V_{\text {ref }}$ and $P$ for the constant $\mathrm{L}$ coil current of $I_{\mathrm{L}}=7 \mathrm{~A}$, and constant magnet position of $z=0 \mathrm{~cm}$, which showed good agreement with (43).

Finally we compare the levitation condition with the stability analysis. Circles in Fig. 13 show minimum $D$ values needed for stable levitation for various $P$ values. We found that levitation was realized when $6.6 \times 10^{-3} \leq D$ and $4.1 \leq P$. When $P$ was below this value, the magnet was not levitated stably with any $D$ value. These values showed fairly good

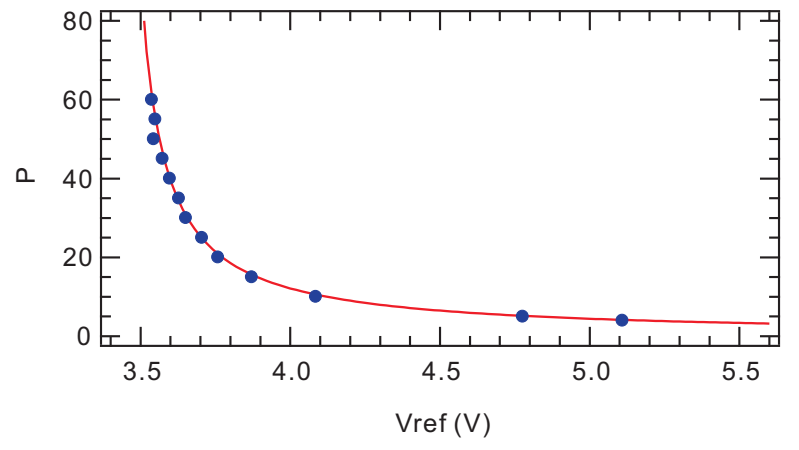

FIG. 12. Set of $V_{\text {ref }}$ and $P$ values that realized stable levitation of the magnet at $z=0 \mathrm{~cm}$ (circles) and the plot of (43) where $\beta=1$ (solid line).

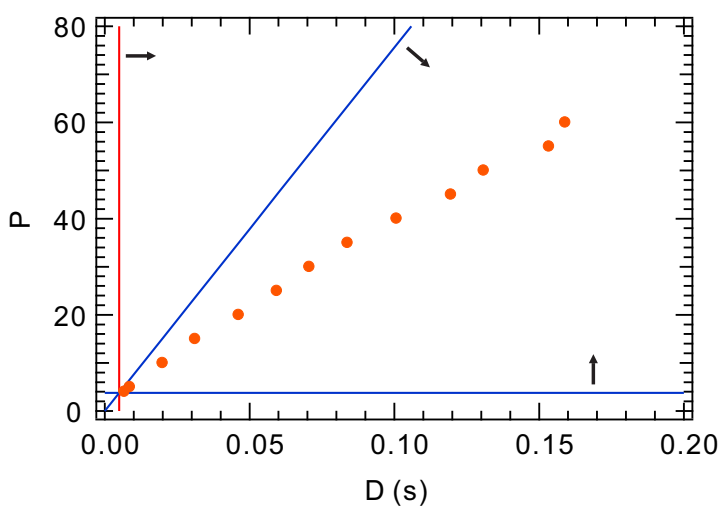

FIG. 13. Comparison of the levitation experiment and stability conditions. Minimum values of $D$ required for the stable levitation (see main text) are plotted as circles for different $P$ values. Numerically obtained stability conditions of (41) (red line) and (42) (blue lines) are also plotted (the arrows indicate conditions to satisfy these equations) for comparison around the measurement region (near the origin of Fig. 10).

agreement with (41) and (42), in spite of the use of a rather simplified analysis model. When the $P$ and $D$ values were close to the stability condition lines in the figure, the magnet behavior was irregular. The magnet position was often stable for a very short time, but eventually vertical instability grew, which was sometimes stabilized for another short time and sometimes not. Such a non-reproductive behavior might be caused by magnetic field errors and rotation of the magnet with small structural and field asymmetries, as well as by the effects of electromagnetic noise in the experiment environment. As a criteria for determination of stability in this figure, we judged that the system is stable when the magnet was levitated for more than $10 \mathrm{~s}$ without significant fluctuations. When the $D$ value was typically two times larger than the critical value expected by (42), the magnet was stably levitated without time constraints, as shown in Fig. 11. 

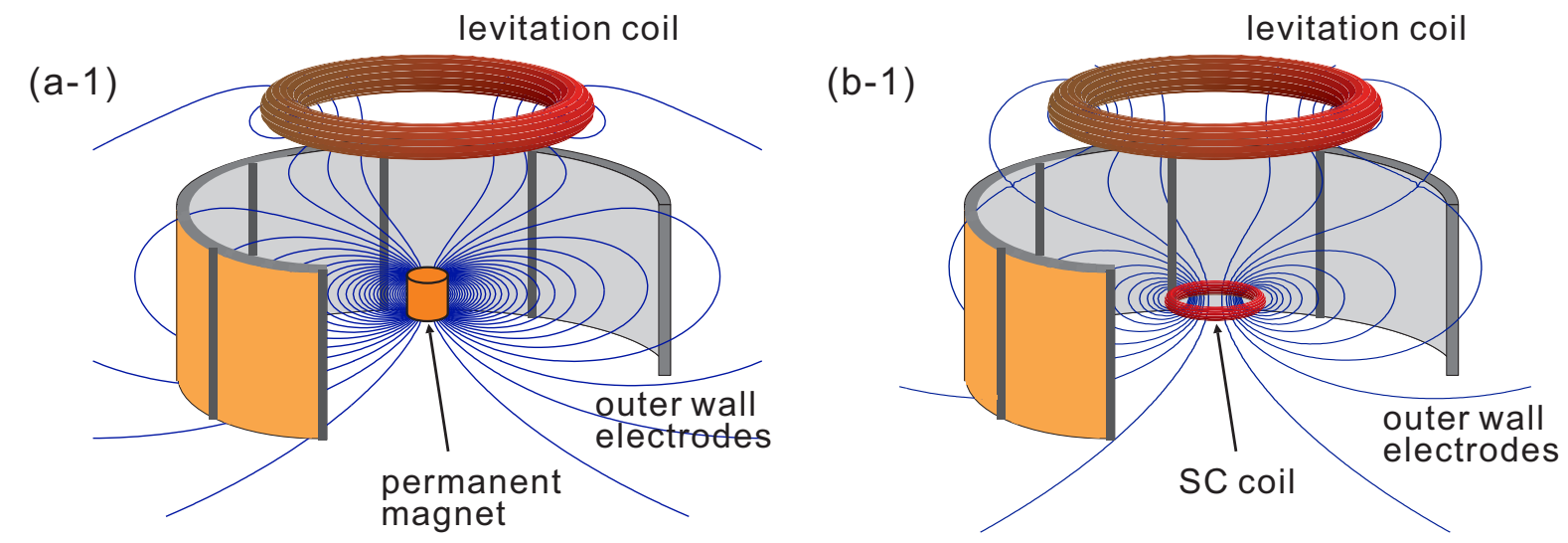

$(a-2)$
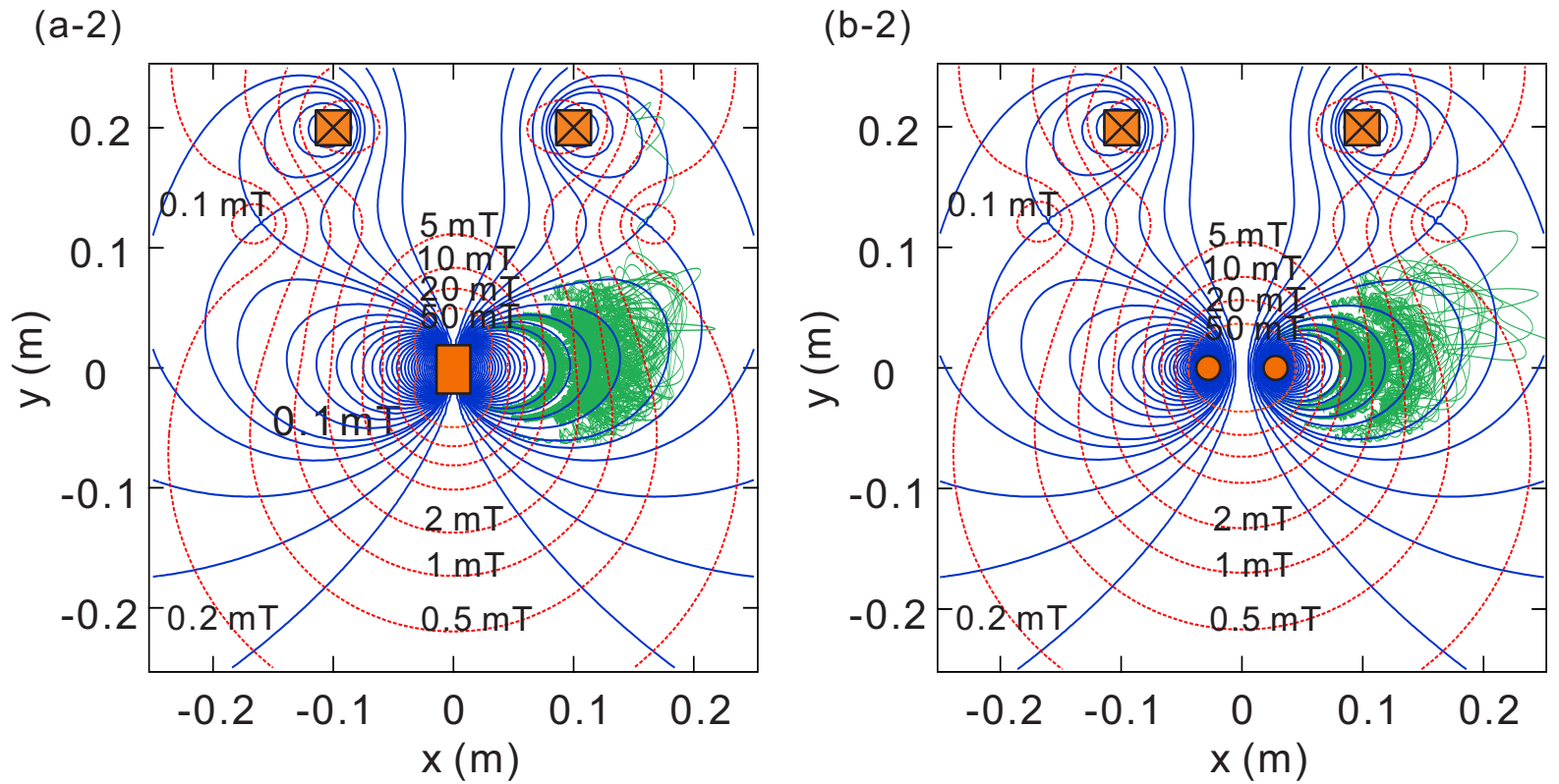

FIG. 14. (1) The schematic views and (2) magnetic configurations of a dipole field trap (a) with a permanent magnet and (b) with a superconducting coil. Green lines (2) show examples of positron orbits projected onto the $r-z$ plane.

\section{TRAPPING PROPERTIES IN PERMANENT MAGNET AND LEVITATED DIPOLE GEOMETRIES}

As demonstrated in the prototype APEX experiment at the NEPOMUC slow positron facility [29], in a dipole magnetic field configuration generated by a permanent magnet, charged particles including positrons are confined for a fairly long time. Because the field lines of a permanent magnet intersect the magnet surface, as shown in Fig. 14 (a-1), however, the upper limit of the trapping time may be set by recombination loss at the magnet surface caused by the collisional transport of particles into the loss cone of the magnetic mirror. In real experiments, it is not straightforward to predict the trapping properties of a dipole field trap because various parameters, including the injection conditions, vacuum and remaining gas species, and the electric and magnetic symmetry of the system in relation to the conservation of canonical angular momentum. In the prototype APEX experiment with a moderate vacuum condition, it was found that the loss cone effects caused by collisions with neutral molecules was an important loss channel of positrons [29]. For single-component nonneutral plasmas, such a loss channel on the magnet surface may be suppressed by applying a DC voltage on the magnet so that trapping electric fields are generated. However, when the trap is asymmetric, for example because of its injection $\mathbf{E} \times \mathbf{B}$ plates located near the trapping region of the APEX experiment [28], bias voltage on the magnet generates asymmetric electric fields that break the conservation of canonical angular momentum of a trapped particle. This can cause a significant degradation of the confinement properties of the trap. Furthermore, when we try to confine multi-component plasmas including electrons and positrons, we cannot apply such bias voltage on the magnet in order to avoid the mirror loss of both of particles.

The particle decay caused by the transport of particles into the loss cone is reduced by replacing the permanent magnet with a levitated superconducting (SC) ring coil in the trap, as shown in Fig. 14, which is planned to be used in the APEX 
experiment. In contrast to those of a permanent magnet, the field lines of a SC coil are closed around the coil, which can greatly reduce the loss of particles compared to the case with a permanent magnet. Here we compare the trapping properties of dipole field configurations generated by a permanent magnet and a SC coil by numerically calculating the particle orbit in these geometries in order to confirm the expected improvement in confinement in the presence of pitch angle scattering elastic collisions. We assume configurations shown in Fig. 14. When the trap has a levitation coil, the variation of the magnetic fields, especially the appearance of the separatrix, greatly modifies the trapping properties. In order to remove such effects in the comparison and to investigate the variation only caused by the replacement of the permanent magnet with the SC coil, we used levitated trapping geometries for both the permanent magnet and SC coil cases. In Fig. 14 (a), a permanent magnet of diameter $28 \mathrm{~mm}$, height $40 \mathrm{~mm}$, and surface field strength $B=0.6 \mathrm{~T}$ is placed at the equator of the trap. These parameters are same as those of a magnet used in the prototype APEX experiment [26]. In (b) of the figure, the magnet is replaced with a SC coil with the same magnetic moment, which is generated by a ring current of $9.8 \mathrm{kA}$ and diameter $56 \mathrm{~mm}$. The magnet and SC coil are levitated by a pulling force between the lifting coil located in the upward direction, as investigated in the previous sections.

In the orbit analysis, we use the parameters of the slow positron beam supplied at the open beam port of NEPOMUC. Namely, positrons with parallel kinetic energy of $5 \mathrm{eV}$ with an energy spread of $1 \mathrm{eV}$ and a perpendicular energy spread of $1 \mathrm{eV}$ were injected from positions near the equator of the trap at $r=8 \mathrm{~cm}$. The initial positions of the particles were distributed with a full width half maximum of $5 \mathrm{~mm}$. Both the energy spreads and spatial position were given as a randomlysampled Gaussian distribution. Optimization of efficient injection of particles [28] into the SC dipole field trap is beyond the scope of this study, although this is an important issue to be studied in future work. The orbit of 1000 particles were calculated with the Bunemann-Boris particle pusher algorithm [46] in the magnetic fields shown in the figure including the effects of neutral collisions. Because the kinetic energy of positrons is quite low, here we considered only random elastic collisions with a constant mean collision frequency $v_{\text {ne }}$. Due to the variation of guiding center position caused by the collisions, some particles are lost after being scattered into the loss cone of magnet or onto field lines that intersect the SC coil. We used the surface shape of the magnet as described above, and that of the SC coil as a torus with minor radius $10 \mathrm{~mm}$ and major radius $28 \mathrm{~mm}$. We also assumed a chamber wall at $r=250 \mathrm{~mm}$ as another loss channel of positrons. When positrons reached these structures, we judged that they are lost and stopped tracking the orbit in the calculation.

Figure 15 shows the fraction of particles remaining in the trap as a function of time when the mean neutral collision frequency was $v_{\text {ne }}=1 \times 10^{-3} f_{\text {gyro }}$, where $f_{\text {gyro }}$ is the mean cyclotron or gyro-frequency. Here the fraction of particles at $t$ is defined as the ratio of positrons that are not lost by annihilation on the magnet and coil surfaces or chamber wall at $t$ after injection at $t=0$. The typical gyrofrequency of a positron in

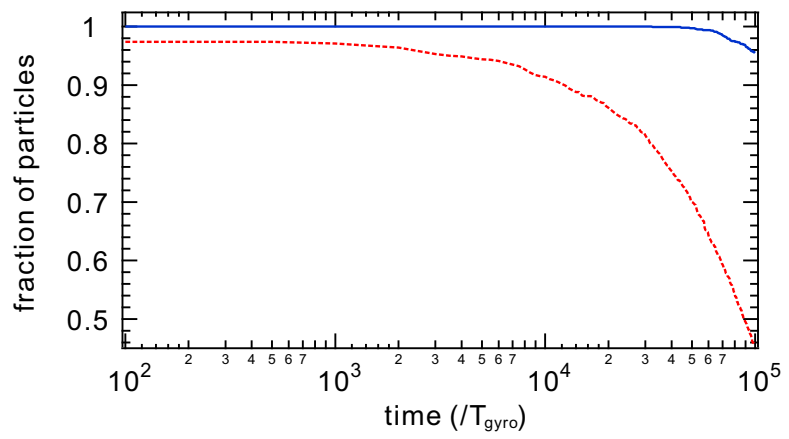

FIG. 15. Fraction of particles remaining in the trap as a function of time in levitated SC coil (solid line) and permanent magnet (dashed line) configurations when the mean neutral collision frequency is $v_{\text {ne }}=1 \times 10^{-3} f_{\text {gyro }}$.

the trapping region is $f_{\text {gyro }}=1 / T_{\text {gyro }}=100 \mathrm{MHz}$. Examples of positron orbits (although particle drift toroidally, the orbits shown are projected onto the r-z plane in the right half of each Fig. 14 (a-2) and (b-2)) clearly show variation of the guiding center caused by neutral collisions. In the total calculation time of $10^{5} T_{\text {gyro }}$, a typical positron makes approximately 100 toroidal rotations due to the curvature and grad-B drift in the dipole magnetic field. In the permanent magnet configuration, as shown by the dashed line in Fig. 15, when particles are initially located in the loss cone, they are promptly lost by hitting the magnet. In the present conditions, the fraction of such positrons was $2.6 \%$. A considerable fraction of positrons are transported into the loss cone and are lost on the magnet surface when neutral collisions affects the positron orbit after $t \sim 1 \times 10^{3} T_{\text {gyro }}$. In contrast, in the geometry of SC coil this loss channel is greatly reduced, as shown by the solid line in the figure. Although there is no loss cone for the SC coil configuration, some of the magnetic field lines intersect the SC coil surface and chamber wall, as shown in Fig. 14. Because of the radial transport of particles into such regions caused by neutral collisions, positrons are gradually lost after $t \sim 4 \times 10^{4} T_{\text {gyro }}$ in the present calculation conditions.

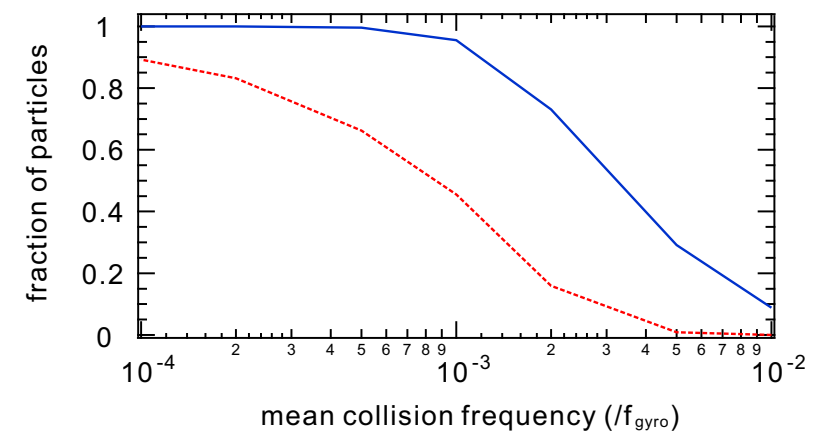

FIG. 16. Fraction of particles at $t=1 \times 10^{5} T_{\text {gyro }}$ in a levitated SC coil (solid line) and permanent magnet (dashed line) configurations for various $v_{\mathrm{ne}}$ values.

For different $v_{\text {ne }}$ values between $10^{-4} f_{\text {gyro }}$ and $10^{-2} f_{\text {gyro }}$, 
the fraction of positrons at $t=10^{5} T_{\text {gyro }}$ is plotted in Fig. 16 . The trapping properties are strongly affected by the neutral collisions. In the permanent magnet trap, where particles are continuously transported into the loss cone, the collision effects are not negligible even at $v_{\text {ne }}=10^{-4} f_{\text {gyro }}$ (the dashed line in the figure). Most of injected positrons are lost at $t=10^{5} T_{\text {gyro }}$ when $v_{\text {ne }}$ is higher than $5 \times 10^{-3} f_{\text {gyro }}$. By replacing the permanent magnet with the $\mathrm{SC}$ coil, as shown by the solid line in the figure, there is a drastic improvement of the trapping properties. While considerable ratio of particles are lost when $1 \times 10^{-3}<v_{\text {ne }}$ because of collisional radial transport toward the SC coil surface and chamber wall, the fraction of positrons of this new geometry exceeds that of the permanent magnet in the entire $v_{\text {ne }}$ range. Although the $v_{\text {ne }}$ values in the present calculation is not necessarily relevant to the actual experiment due to the calculation time limitation, we can expect the improved confinement properties in the SC dipole compared with the permanent magnet configuration by properly choosing the experimental conditions.

\section{CONCLUSION}

To conclude, we developed a feedback-controlled magnetic levitation system to be used for the levitation of a superconducting dipole field coil in the APEX pair-plasma project. The properties of the levitation system were investigated using a mock-up experiment with a permanent magnet. Comparison of the experimental results showed fairly good agreement with numerical analysis of the equilibrium and stability of the mag- net motion. These results provide a concrete basis for the design of a compact levitated dipole experiment for the APEX project. Together with the experimental results, in this paper, we also presented a practical review on the levitation physics needed for the construction of a feedback-controlled magnetic levitation system for a charged particle trap. In order to estimate the trapping properties of charged particles in the dipole field trap by replacing the permanent magnet with a levitated superconducting coil, especially to investigate the effects of the reduction of loss cone, we analyzed the orbit of slow positrons in both of the trapping geometries. Assuming slow radial transport of particles across magnetic field lines, which is realized by collisions with neutral particles in the present study, the comparison calculation showed the possibility of improved confinement properties in the new superconducting dipole field configuration. These results suggest that a levitated superconducting dipole trap is potentially applicable to a compact and low-cost trapping geometry for various charged particles in atomic and non-neutral plasmas, and especially for the electron-positron plasmas in the APEX experiment.

\section{ACKNOWLEDGMENT}

The authors thank J. Morikawa, Prof. Y. Ogawa, Prof. Z. Yoshida, Prof. Yanagi, Dr. Y. Yano, and the PAX/APEX group members for valuable comments and discussions. The PAX and APEX project is supported by ERC, DFG, IPP, UCSD, and NIFS. This work was supported by JSPS KAKENHI Grant No. 25707043 and 16KK0094.
[1] S. Yoshikawa, Nucl. Fusion 13, 433 (1973).

[2] Z. Yoshida, Y. Ogawa, J. Morikawa, S. Watanabe, Y. Yano, S. Mizumaki, T. Tosaka, Y. Ohtani, A. Hayakawa, and M. Shibui, Plasma Fusion Res. 1, 008 (2006).

[3] S. M. Krimigis, T.P. Armstrong, W. I. Axford, C. O. Bostrom, C. Y. Fan, G. Gloeckler, L. J. Lanzerotti, E. P. Keath, R. D. Zwickl, J. F. Carbary, and D. C. Hamilton, Science 206, 977 (1979).

[4] S. M. Mahajan and Z. Yoshida, Phys. Rev. Lett. 81, 4863 (1998).

[5] Z. Yoshida and S. M. Mahajan, Phys. Rev. Lett. 88, 095001 (2002).

[6] Z. Yoshida et al., Proceedings of 17th IAEA Fusion Energy Conference (IAEA), IAEACN-69/ICP/10 (R), (1998).

[7] H. Saitoh, Z. Yoshida, C. Nakashima, H. Himura, J. Morikawa, and M. Fukao, Phys. Rev. Lett. 92, 255005 (2004).

[8] Y. Ogawa, J. Morikawa, K. Uchijima, Y. Hosaka, C. Kawai, K. Ibano, T. Mito, N. Yanagi, K. Natsume, Y. Terazaki, M. Iwakuma, A. Tomioka, and S. Nose, Plasma and Fusion Res. 9, 1405014 (2014).

[9] Z. Yoshida, H. Saitoh, J. Morikawa, Y. Yano, S. Watanabe, and Y. Ogawa, Phys. Rev. Lett. 104, 235004 (2010).

[10] M. Nishiura, Y. Kawazura, Z. Yoshida, N. Kenmochi, Y. Yano, H. Saitoh, M. Yamasaki, T. Mushiake, A. Kashyap, and N. Takahashi, Nucl. Fusion 57, 086038 (2017).
[11] A. Hasegawa, Comments Plasma Phys. Control. Fusion 11, 147 (1987).

[12] A. C. Boxer, R. Bergmann, J. L. Ellsworth, D. T. Garnier, J. Kesner, M. E. Mauel, and P. Woskov, Nat. Phys. 6, 207 (2010).

[13] D. T. Garnier, A. K. Hansen, J. Kesner, M. E. Mauel, P. C. Michael, J. V. Minervini, A. Radovinsky, A. Zhukovsky, A. Boxer, J. L. Ellsworth, I. Karim, E. E. Ortiz , Fusion Eng. Design 81, 2371 (2006).

[14] R. C. Davidson, Physics of Nonneutral Plasmas (World Scientific, 2001).

[15] J. R. Danielson, D. H. E. Dubin, R. G. Greaves, and C. M. Surko, Rev. Mod. Phys. 87, 247 (2015).

[16] H. T. Schmidt, R. D. Thomas, M. Gatchell, S. Rosén, P. Reinhed et. al., Rev. Sci. Instrum. 84, 055115 (2013).

[17] Y. Nakano, Y. Enomoto, T. Masunaga, S. Menk, P. Bertier, and T. Azuma, Rev. Sci. Instrum. 88, 033110 (2017).

[18] M. Aramaki, Y. Sakawa, and T. Shoji, Jpn. J. Appl. Phys. 39, L246 (2000).

[19] H. Himura, Nucl. Instrum. Methods Phys. Res., A 811, 100 (2016).

[20] Z. Yoshida, Y. Ogawa, J. Morikawa, H. Himura, S. Kondoh, C. Nakashima, S. Kakuno, M. Iqbal, F. Volponi, N. Shibayama, and S. Tahara, AIP Conf. Proc. 498, 397 (1999).

[21] T. Sunn Pedersen and A. H. Boozer, Phys. Rev. Lett. 88, 205002 (2002). 
[22] T. Sunn Pedersen, J. R. Danielson, C. Hugenschmidt, G. Marx, X. Sarasola, F. Schauer, L. Schweikhard, C. M. Surko, and E. Winkler, New J. Phys. 14, 035010 (2012).

[23] H. Higaki, C. Kaga, K. Fukushima, H. Okamoto, Y. Nagata, Y. Kanai, and Y. Yamazaki, New J. Phys. 19, 023016 (2017).

[24] Hui Chen, F. Fiuza, A. Link, A. Hazi, M. Hill, D. Hoarty, S. James, S. Kerr, D. D. Meyerhofer, J. Myatt, J. Park, Y. Sentoku, and G. J. Williams, Phys. Rev. Lett. 114, 215001 (2015).

[25] G. Sarri, K. Poder, J. M. Cole, W. Schumaker, A. Di Piazza, B. Reville, T. Dzelzainis, D. Doria, L. A. Gizzi, G. Grittani, et. al., Nat. Commun. 6, 6747 (2015).

[26] H. Saitoh, J. Stanja, E. V. Stenson, U. Hergenhahn, H. Niemann, T. Sunn Pedersen, M. R. Stoneking, C. Piochacz, and C Hugenschmidt, New J. Physics 17, 103038 (2015).

[27] C. Hugenschmidt, C. Piochacz, M. Reiner, and K. Schreckenbach, New J. Physics 14, 055027 (2012).

[28] E. V. Stenson, S. Niß1, U. Hergenhahn, J. Horn-Stanja, M. Singer, H. Saitoh, T. Sunn Pedersen, J. R. Danielson, M. R. Stoneking, M. Dickmann, and C. Hugenschmidt, Phys. Rev. Lett. 121, 235005 (2018).

[29] J. Horn-Stanja, S. Niß1, U. Hergenhahn, T. Sunn Pedersen, H. Saitoh, E. V. Stenson, M. Dickmann, C. Hugenschmidt, M. Singer, M. R. Stoneking, and J. R. Danielson, Phys. Rev. Lett. 121, 235003 (2018).

[30] J. Stanja, U. Hergenhahn, H. Niemann, N. Paschkowski, T. Sunn Pedersen, H. Saitoh, E. V. Stenson, M. R. Stoneking, C. Hugenschmidt, and C. Piochcz, Nucl. Instrum. Meth. Phys. Res. A 827, 52 (2016).

[31] J. Horn-Stanja, E. V. Stenson, M. R. Stoneking, M. Singer, U. Hergenhahn, S. Niß1, H. Saitoh, T. Sunn Pedersen, M. Dickmann, C. Hugenschmidt, and J. R. Danielson, Plasma Res. Exp. 2, 015006 (2020).

[32] S. Earnshaw, Trans. Camb. Phil. Soc. 7, 97 (1842).
[33] V. Kelvin Neil and Richard K. Cooper, Rev. Sci. Instrum. 40, 295 (1969).

[34] C. H. Woods, R. K. Cooper, V. K. Neil, and C. E. Taylor, J. Appl. Phys. 41, 3295 (1970).

[35] J. File, G. D. Martin, R. G. Mills, and K. E. Wakefield, J. Appl. Phys. 42, 6 (1971).

[36] I. Echeverría and M. A. Rubio, Rev. Sci. Instrum. 66, 3931 (1995).

[37] S. Baer, M. A. B. Andrade, C. Esen, J. C. Adamowski, G. Schweiger, and A. Ostendorf, Rev. Sci. Instrum. 82, 105111 (2011).

[38] J. P. Houlton, M. L. Chen, M. D. Brubaker, K. A. Bertness, and C. T. Rogers, Rev. Sci. Instrum. 89, 125107 (2018).

[39] J. Morikawa, D. Ozawa, Y. Ogawa, N. Yanagi, S. Hamaguchi, and T. Mito, Jpn. J. Appl. Phys. 40, L1029 (2001).

[40] N. Yanagi, J. Morikawa, T. Mito, Y. Ogawa, S. Hamaguchi, H. Chikaraishi, M. Iwakuma, T. Ueno, I. Itoh, S. Nose, and S. Fukui, IEEE Trans. App. Superconductivity 12, 948 (2002).

[41] Y. Ogawa, J. Morikawa, K. Ohkuni, H. Nihei, D. Hori, S. Yamakoshi, T. Goto, T. Mito, N. Yanagi, M. Iwakuma, and T. Uede, TEION KOGAKU, J. Cryo. Soc. Jpn. (in Japanese) 39, 175 (2004).

[42] J. Morikawa, K. Ohkuni, D. Hori, S. Yamakoshi, T. Goto, Y. Ogawa, N. Yanagi, and T. Mito, TEION KOGAKU, J. Cryo. Soc. Jpn. (in Japanese) 39, 209 (2004).

[43] Y. Yano, Z. Yoshida, Y. Ogawa, J. Morikawa, and H. Saitoh, Fusion Eng. Design 85, 641 (2010).

[44] M. R. Stoneking, H. Saitoh, M. Singer, E. V. Stenson, J. HornStanja, T. Sunn Pedersen, S. Niß1, U. Hergenhahn, N. Yanagi, C. Hugenschmidt, M. Dickmann, J. R. Danielson, and C. M. Surko, AIP Conf. Proc. 1928, 020015 (2018).

[45] A. Hurwitz, Math. Ann. 46, 273 (1895).

[46] H. Qin, S. Zhang, J. Xiao, J. Liu, Y. Sun, and W. M. Tang, Phys. Plasmas 20, 084503 (2013). 